\section{Krebs von den Lungen-6, a promising marker in evaluating the severity of interstitial lung disease in Egyptian rheumatoid arthritis patients}

\author{
Rasha M. Hammoda ${ }^{1}$, Haitham Salah-EIDin ${ }^{2}$, \\ Nermin H. EL-Gharbawy ${ }^{3}$ and Dina Ragab ${ }^{4}$
}

\author{
${ }^{1}$ Department of Internal Medicine \& Rheumatology, Faculty of \\ Medicine, Ain Shams University, Cairo, Egypt. \\ ${ }^{2}$ Department of Chest, Faculty of Medicine, Ain Shams \\ University, Cairo, Egypt. \\ ${ }^{3}$ Department of Physical Medicine, Rheumatology \& \\ Rehabilitation, Faculty of Medicine, Ain Shams University, \\ Cairo, Egypt.
}

The Egyptian Journal of Immunology Volume 28 (4), 2021: 241-249. www.Ejimmunology.org

\begin{abstract}
Krebs von den Lungen- $6(\mathrm{KL}-6)$ is one of the mucins associated with interstitial lung disease. We aimed to assess the value of KL- 6 as a marker for detecting the presence of interstitial lung disease in Egyptian rheumatoid arthritis patients and to evaluate its ability to assess severity in different grades of interstitial lung disease. The study included 89 rheumatoid arthritis patients; 64 patients with interstitial lung disease and 25 patients without interstitial lung disease. Serum levels of KL- 6 were assessed using enzyme linked immunosorbent assay. Levels of KL-6 were higher in patients with interstitial lung disease compared to patients without interstitial lung disease $(P<0.001)$. KL- 6 levels were significantly higher in grade 4 patients than those in grades 1 and 2. Also, KL-6 levels were significantly higher in grade 3 patients than those in grades 1 and 2 . $\mathrm{KI}-6$ levels were also higher in grade 2 patients compared to grade 1 patients. Finally, no difference was observed between grade 4 patients and grade 3 patients. KL- 6 levels were significantly higher in usual interstitial pneumonia pattern compared other patterns $(P=0.015)$. In conclusion, KL- 6 is a potential circulating biomarker that may have a substantial role in detecting the presence and evaluating the severity of interstitial lung disease among rheumatoid arthritis patients.
\end{abstract}

Keywords: interstitial lung disease; Krebs von den Lungen-6; rheumatoid arthritis.

Date received: 23 August 2021; accepted: 27 September 2021

\section{Introduction}

Rheumatoid arthritis (RA) is a systemic chronic inflammatory disease of autoimmune nature, affecting about $0.5-1 \%$ of worldwide population. Despite of the available therapeutic modalities for RA, the disease still had substantial morbidity and mortality. ${ }^{1}$ The disease involves both articular and extraarticular manifestations. Rheumatoid arthritis can be associated with a wide variety of extraarticular manifestations and comorbidities. Extra-articular affection occurs in more than $35 \%$ of RA patients and can contribute to the 
physical disability and psychological morbidity of the disease leading to reduced quality of life, requirement for more intense treatment approaches, higher direct costs and indirect social cost of the disease. Extra-articular manifestations can range in severity from nonlife-threating conditions like subcutaneous rheumatoid nodules to severe conditions like vasculitis, cardiac affection, pachymeningitis and lung affection. ${ }^{2}$ Although cardiovascular disease remains the most common cause of RAassociated mortality, pulmonary involvement is also a major contributor to RA-associated mortality, accounting for nearly $10-20 \%$ of all deaths. ${ }^{3}$ Interstitial lung disease (ILD) represents the second leading cause of death in RA patients. $^{4}$

Interstitial lung disease is a common manifestation of pulmonary involvement in several connective tissue diseases including RA, ${ }^{5}$ systemic lupus erythematosus, ${ }^{6}$ Systemic Sclerosis, $^{7}$ Sjogren's syndrome ${ }^{8,9}$ and inflammatory myositis. ${ }^{10}$ ILD is characterized by parenchymal inflammation and/or fibrosis of the interstitium, alveoli or terminal bronchioles. ${ }^{11}$ ILD is the most common form of RA associated pulmonary diseases and it is characterized by diffuse pulmonary parenchymal inflammation and interstitial fibrosis. ILD occurrence may precede articular manifestations. Moreover, $6-15 \%$ of RA patients have a risk of developing ILD, and the risk increases with prolonged RA duration. ${ }^{12}$ Death risk was found to be 3 times higher in RA patients with ILD compared to RA patients who do not have ILD. Given the high mortality associated with ILD, early diagnosis and appropriate intervention is critical. ${ }^{13}$

Diagnosis of ILD in RA patients depends on high-resolution computed tomography (HRCT) and pulmonary function tests (PFT) in addition to the clinical symptoms and clinical examination. HRCT is an important tool for diagnosing different ILD abnormalities in RA patients. HRCT even has the ability to identify subclinical cases before clinical presentation. ${ }^{14}$

The presence of blood biomarkers, that could have an efficiency comparable to HRCT in detecting ILD associated with RA, would greatly facilitate the disease diagnosis and allow for early aggressive intervention prior to disease progression. ${ }^{15}$ Krebs von den Lungen- 6 (KL-6) is one of the mucins associated with respiratory cellular membranes. It is mainly expressed in alveolar type II pneumocytes and epithelial cells lining the respiratory bronchioles. KL-6 is an indicator of alveolar damage. During lung injury, KL-6 significantly increases due to alveolar epithelial injury where KL- 6 becomes secreted in the bloodstream through the damaged basement membrane. ${ }^{16}$ Hence, in this study we aimed to assess the value of KL- 6 as a marker for detecting the presence of ILD in RA patients and to evaluate its ability in assessing the ILD severity.

\section{Materials and Methods}

\section{Patients}

Sixty-four RA patients with ILD were enrolled in the study. Twenty-five age and sex matched RA patients with no evidence of ILD by HRCT were enrolled as a comparator group. Patients with ILD were further classified into 4 subgroups according to the grade of ILD by HRCT.

RA patients were diagnosed according to the American College of Rheumatology and European League against Rheumatism (ACR/EULAR) 2010 classification criteria. ${ }^{17,} 18$ Patients were recruited from the outpatient Rheumatology clinic and inpatient Department of Rheumatology, Ain Shams University Hospital. The study protocol was reviewed and approved by The Ethical Committee, Faculty of Medicine, Ain Shams University (FMASU R 99, 2020). Each patient provided a written informed consent prior to enrollment in the study.

\section{Sample collection}

Two venous blood samples were obtained from each participant. The first sample $(4 \mathrm{ml})$ was collected into a serum separating tube. The sample was allowed to clot then centrifuged at $2000 \mathrm{~g}$ for $20 \mathrm{~min}$, serum was the separated and stored $-20{ }^{\circ} \mathrm{C}$ until used for measurement of Creactive protein (CRP), rheumatoid factor (RF), anti-cyclic citrullinated antibody (Anti-CCP) and KL- 6 levels. The second sample $(2 \mathrm{ml})$ was collected on $3.2 \% \mathrm{Na}$ citrate vacutainer tube 
and used for assessment of erythrocyte sedimentation rate (ESR).

\section{Laboratory tests}

- Serum levels of CRP and RF were determined using Cobas c6000 analyzer (Roche diagnostics, Switzerland). Serum levels of Anti-CCP were determined using Cobas e411 analyzer (Roche diagnostics, Switzerland). ESR was determined using the Westergren method.

- Serum levels of KL-6 were assayed using commercially available enzyme-linked immunosorbent assay kit supplied by MyBioSource, San Diego, USA, according to the manufacturer's instructions. Briefly, $50 \mu \mathrm{l}$ of each standard and sample were added to the corresponding wells, then $100 \mu$ of the HRPconjugate were added to each well. The plate was then incubated at $37^{\circ} \mathrm{C}$ for 60 minutes followed by washing of the wells 4 times. Then, $50 \mu$ of Chromogen A and $50 \mu$ of chromogen B were added to each well followed by incubation at $37^{\circ} \mathrm{C}$ for 15 minutes away from light, after which $50 \mu$ of the stop solution were added and the optical density was read at 450nm using Stat Fax 2100 microplate reader (Awareness Technology, USA). A standard curve was plotted using the standards' readings, and concentrations of the samples were deduced from the curve.

\section{HRCT Evaluation}

Chest HRCT was done for all patients within 48 hours of blood sample withdrawal. HRCT was done in supine position at end inspiration without contrast enhancement. Images were reviewed by a radiologist and a chest consultant with inter-observer agreement. ILD extent was graded semi-quantitatively by assessing the total CT \% of pulmonary parenchymal abnormality into: (Grade 1: 1-25\%; Grade 2: 26-50\%; Grade 3: $51-75 \%$ and Grade 4: 76$100 \%)$.

\section{Statistical analysis}

Analysis was done using Graph Pad Prism software, version 8.01 (San Diego, CA, USA). Nominal data were expressed as frequency and percentage, association between data was examined using Chi-square test while Fisher's exact test was used when appropriate. Quantitative non-parametric data were presented as median and interquartile range. Groups were compared using Mann-Whitney to compare between two groups and KruskalWallis test to compare between more than two groups and whenever significant a post-hoc test was used for pair-wise comparison. Receiver Operating Characteristic (ROC) curve was plotted to evaluate the performance of KL-6. $P$ value $<0.05$ was considered statistically significant.

\section{Results}

\section{Patients' demographics}

A total of 89 RA patients were enrolled in the study, including 22 males (24.7\%) and 67 females $(67.3 \%)$ with mean age $42.8 \pm 11.4$ years. Of these, 25 RA patients (28.1\%) had no ILD while 64 patients (71.9\%) had ILD. Among the ILD patients, 17 patients had grade I ILD, 28 patients had grade 2 ILD, 15 patients had grade 3 ILD while 4 patients had grade 4 ILD. A usual interstitial pneumonia (UIP) pattern of ILD was found in 29 patients while 35 patients had other ILD patterns. Clinical and demographic characteristic of studied patients are shown in Table 1. Patients with ILD had significantly higher ESR and KL-6 levels, however, no significant difference was observed between both patients groups regarding age, gender, CRP levels, number of patients positive for RF and number of patients positive for anti-CCP. Also, no difference was found regarding the types of received medications. 
Table 1. Clinical and demographic characteristics of patients.

\begin{tabular}{|c|c|c|c|}
\hline & RA $(n=25)$ & RA+ILD $(n=64)$ & $P$-value \\
\hline Age in years, mean $\pm S D$ & $43.9 \pm 9.6$ & $47.7 \pm 11.8$ & NS \\
\hline Gender, M/F & $7 / 18$ & $15 / 49$ & NS \\
\hline ILD HRCT grade (\%) & & $\mathrm{n}(\%)$ & \\
\hline Grade 1 (1\%-25\%) & & $17(26.6 \%)$ & \\
\hline Grade 2 (26\%-50\%) & & $28(43.8 \%)$ & \\
\hline Grade 3 (51\%-75\%) & & $15(23.4 \%)$ & \\
\hline Grade 4 (76\%-100\%) & & $4(6.2 \%)$ & \\
\hline ILD pattern & & $\mathrm{n}(\%)$ & \\
\hline UIP & & $29(45.3 \%)$ & \\
\hline Non-UIP & & $35(54.7 \%)$ & \\
\hline Medications & $\mathrm{n}(\%)$ & $\mathrm{n}(\%)$ & \\
\hline Corticosteroid & $25(100 \%)$ & $63(98.4 \%)$ & \\
\hline Methotrexate & $23(92 \%)$ & $3(4.7 \%)$ & \\
\hline Leflunomide & $6(24 \%)$ & 0 & \\
\hline Hydroxychloroquine & $19(76 \%)$ & $48(75 \%)$ & \\
\hline Cyclophosphamide & 0 & $35(54.7 \%)$ & \\
\hline Mycophenolate Mofetil & 0 & $20(31.3 \%)$ & \\
\hline Azathioprine & 0 & 9 (14.1\%) & \\
\hline Biological & $5(20 \%)$ & 0 & \\
\hline CRP mg/L, median (IQR) & $15.0(9.5-27.5)$ & $18.5(12.0-35.7)$ & NS \\
\hline ESR $\mathrm{mm} / \mathrm{h}$, median (IQR) & $34(13-55)$ & $65(52-76)$ & $<0.001$ \\
\hline Positive RF, n (\%) & $21(84 \%)$ & $55(85.9 \%)$ & NS \\
\hline Positive Anti-CCP, n (\%) & $20(80 \%)$ & $56(87.5 \%)$ & NS \\
\hline KL-6 U/mL, median (IQR) & 324 (281-418) & $1235(1100-1455)$ & $<0.001$ \\
\hline
\end{tabular}

Higher KL-6 levels in RA patients with ILD

KL-6 levels were significantly higher in RA patients with ILD than RA patients without ILD (median:1235 U/mL versus $324 \mathrm{U} / \mathrm{mL} ; P<0.001$ ) (Figure $1 \mathrm{~A})$. Regarding the performance of KL-6 in discriminating between patients with ILD from patients without ILD, an ROC curve was plotted, and the best cut-off was $485 \mathrm{U} / \mathrm{mL}$ with a sensitivity of $100 \%$, of specificity $96 \%$, positive predictive value (PPV) of $98.5 \%$, negative predictive value (NPV) of $100 \%$ and efficiency of 98.9\%. The area under the curve (AUC) was 0.999 with $95 \% \mathrm{Cl}$ of $(0.995-1.002)$ (Figure1B and Table 2).
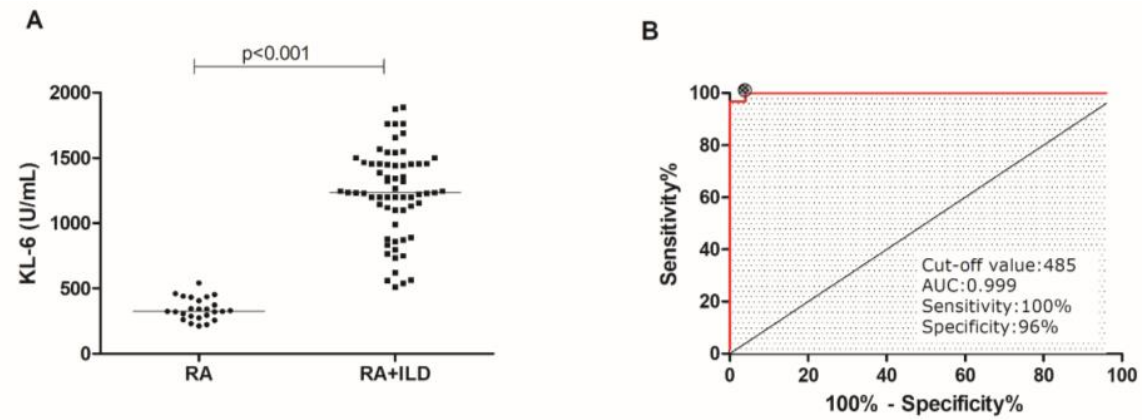

Figure 1. Higher levels of KL-6 in RA patients with ILD (RA+ILD) compared to RA patients without ILD (RA) (median is represented by a horizontal line). B) ROC curve demonstrating the optimal cut-off of $\mathrm{KL}-6$ to detect the presence of ILD in RA patients. 
Table 2. Performance characteristics of KL-6 ROC curve cut-off values.

\begin{tabular}{lcccc}
\hline & ILD vs no ILD & $\begin{array}{c}\text { Grade 1 ILD vs } \\
\text { Grades 2, 3, 4 }\end{array}$ & $\begin{array}{c}\text { Grades 1, 2 ILD vs } \\
\text { Grades 3, 4 }\end{array}$ & $\begin{array}{c}\text { Grades 1,2, 3 ILD vs } \\
\text { Grade4 }\end{array}$ \\
\hline KL-6 cut-off & $485 \mathrm{U} / \mathrm{mL}$ & $940 \mathrm{U} / \mathrm{ML}$ & $1415 \mathrm{U} / \mathrm{mL}$ & $1559 \mathrm{U} / \mathrm{mL}$ \\
\hline AUC & 0.999 & 0.915 & 0.918 & 0.981 \\
$(95 \%$ Cl) & $(0.995-1.002)$ & $(0.815-1.015)$ & $(0.845-0.991)$ & $(0.949-1.014)$ \\
\hline Sensitivity & $100 \%$ & $100 \%$ & $89.5 \%$ & $100 \%$ \\
\hline Specificity & $96 \%$ & $82.4 \%$ & $88.9 \%$ & $93.3 \%$ \\
\hline PPV & $98.5 \%$ & $94 \%$ & $77.3 \%$ & $50 \%$ \\
\hline NPV & $100 \%$ & $100 \%$ & $95.2 \%$ & $100 \%$ \\
\hline Efficiency & $98.9 \%$ & $95.3 \%$ & $89.1 \%$ & $93.8 \%$ \\
\hline
\end{tabular}

AUC: area under the curve; $\mathrm{Cl}$ : confidence interval; ILD: interstitial lung disease; NPV: negative predictive value; PPV: positive predictive value.

Patients were divided according to pattern of ILD into UIP pattern and other patterns (nonUIP). When KL-6 levels were compared between both groups, the levels were significantly higher in these with UIP pattern compared to non-UIP patterns (median: $1443 \mathrm{U} / \mathrm{mL}$ versus $1200 \mathrm{U} / \mathrm{mL}$; $P=0.018$ ) (Figure 2).

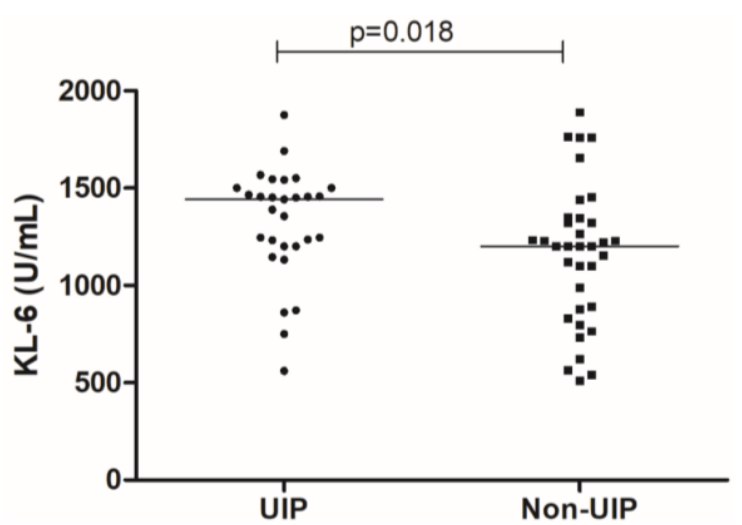

Figure 2. Higher levels of KL-6 in patients with usual interstitial pneumonia (UIP) pattern (median is represented by a horizontal line).

\section{$K L-6$ levels and severity of ILD}

To examine whether levels of KL- 6 in RA patients with ILD were related to grade of ILD, we divided the patients into four groups according to their ILD grade by HRCT and compared the levels of KL-6 between the four groups (Figure $3 \mathrm{~A}$ ). Levels of KL-6 were significantly different between the 4 grades of
ILD $(P<0.001)$. KL-6 levels were significantly higher in grade 4 patients than those in grade 1 and grade 2 ( $P<0.001 ; P<0.05$ respectively). Also, KL-6 levels were significantly higher in grade 3 patients than those in grade 1 and grade $2(P<0.001 ; P<0.05$, respectively). KL- 6 levels were also higher in grade 2 patients compared to grade 1 patients $(P<0.01)$. Finally, KL- 6 levels did not differ between the late stages of ILD; grade 4 patients and grade 3 patients $(P>0.05)$.

ROC curves were plotted to determine the performance of KL-6 in discriminating between different ILD grades (Table 2). For discriminating grades 2, 3 and 4 from grade 1, the best cut-off was $940 \mathrm{U} / \mathrm{mL}$ with a sensitivity of $100 \%$, specificity of $82.4 \%$, PPV of $94 \%$, NPV of $100 \%$ and efficiency of $95.3 \%$ with AUC of 0.915 and $95 \% \quad \mathrm{Cl} \quad(0.815-1.015)$ (Figure 3B). For discriminating grades 3 and 4 from grades 1 and 2 , the best cut-off was $1415 \mathrm{U} / \mathrm{mL}$ with a sensitivity of $89.5 \%$, specificity of $88.9 \%$, PPV of $77.3 \%$, NPV of $95.2 \%$ and efficiency of $89.1 \%$ with AUC of 0.918 and $95 \% \mathrm{Cl}(0.845-0.991)$ (Figure 3C). Finally, for discriminating grades 4 from grades 1, 2 and 3, the best cut-off was $1559 \mathrm{U} / \mathrm{mL}$ with a sensitivity of $100 \%$, specificity of $93.3 \%$, PPV of $50 \%$, NPV of $100 \%$ and efficiency of $93.8 \%$ with AUC of 0.981 and $95 \%$ $\mathrm{Cl}$ (0.949-1.014) (Figure 3D). 


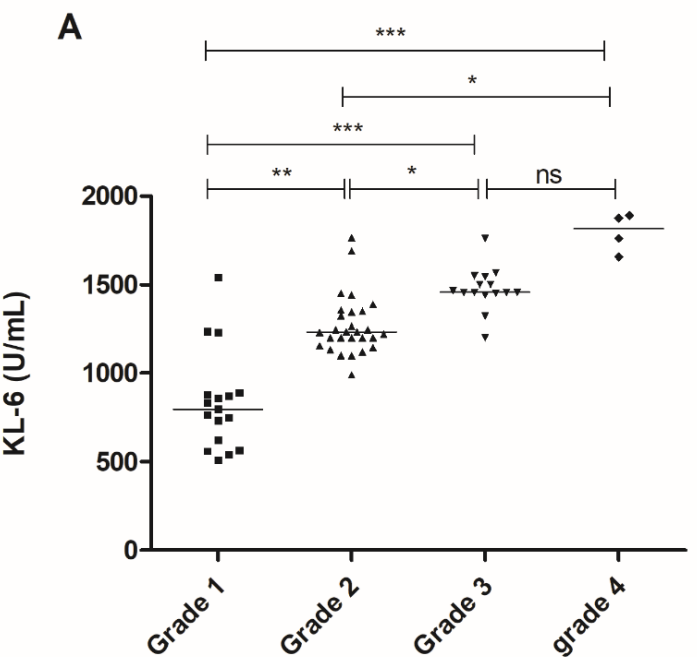

C

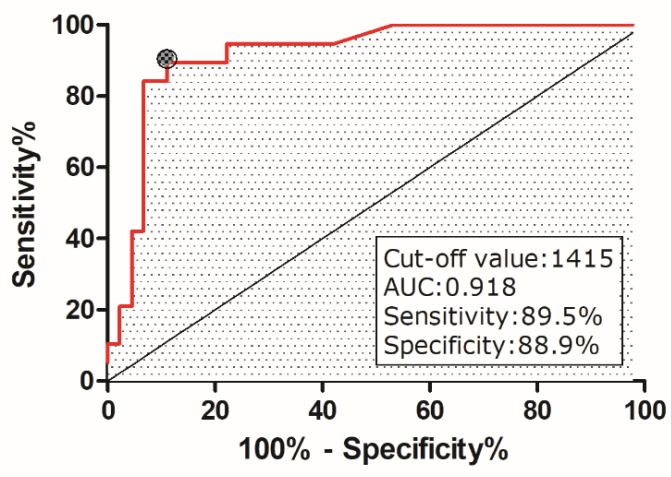

B

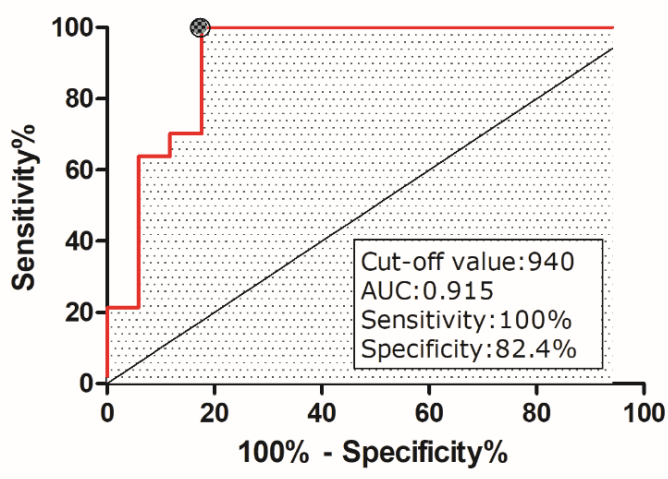

D

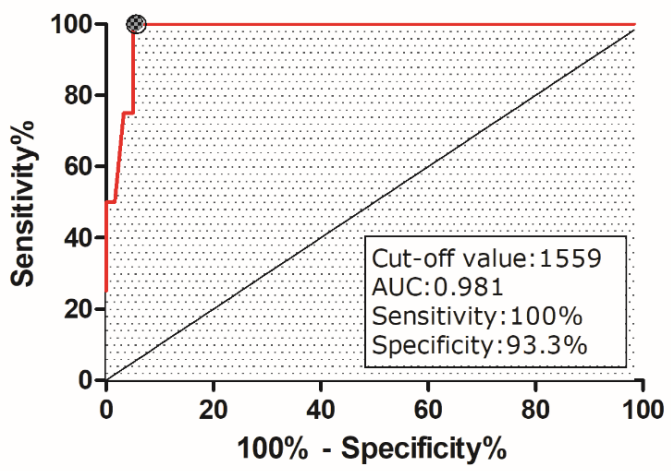

Figure 3. A) Levels of KL- 6 in RA patients with ILD according to the grade of ILD by HRCT (median is represented by a horizontal line). B) ROC curve for discriminating grade 1 ILD from grades 2, 3 and 4 . C) ROC curve for discriminating grades 1 and 2 from grades 3 and 4 ILD. D) ROC curve for discriminating grades 1,2 and 3 from grade 4 ILD. ns: non-significant; ${ }^{*} P<0.05 ;{ }^{* *} P<0.01 ;{ }^{* * *} P<0.001$.

\section{Discussion}

The deterioration of lung functions resulting from ILD impacts the prognosis of patients and leads to increased mortality. Early detection, timely treatment and proper intervention could be critical to improve the clinical outcome and survival of patients with ILD. Currently, diagnosis of ILD mainly depends on HRCT and pulmonary function tests, however these tests have some inherent caveats including the high cost, radiation exposure, poor reproducibility, as well as the incompatibility for critically ill patients. Hence, finding reliable circulating biomarkers may aid in earlier diagnosis and clinical assessment of these cases. ${ }^{19,20} \mathrm{KL}-6$ is being intensively investigated as a circulating biomarker in the context of ILD, ${ }^{21,22}$ ILD associated with connective tissue diseases (CTDILD), ${ }^{16,} \quad 23, \quad 24$ ILD associated with systemic sclerosis $^{19,25}$ and ILD associated with RA. ${ }^{20,26-28}$

In this study, we assessed the levels of KL-6 in 89 RA patients; 25 patients without ILD and 64 patients with ILD and evaluated the performance of KL- 6 in diagnosis of ILD. Results revealed higher levels of serum KL- 6 in RA patients with ILD compared to those without ILD. KL-6 is a mucin-like glycoprotein, it is classified as mucin 1 , cell surface associated (MUC1) and is mainly expressed on type II pneumocytes. During alveolar inflammation, inflammatory cells damage basement membrane and epithelial cells of the alveolar wall and KL-6 is released into blood. Thus, during the inflammatory and regenerative process of ILD, KL-6 increases reflecting alveolar 
epithelial cells damages. ${ }^{20}$ Our findings are consistent with those reported by several studies. Avouac et al., $2020^{20}$ assessed KL-6 levels in 40 RA patients with ILD and 107 RA patients without ILD and reported higher levels of KL-6 in RA patients with ILD compared to RA without ILD. The study included patients from France, Japan and Switzerland. Kinoshita et al. $2004^{26}$ also reported similar results in a study in Japan that included 21 RA patients with ILD and 29 RA patients without ILD. Moreover, Lee et al., 2019 23 assessed KL-6 in CTD-ILD patients in Korea and the study included 549 connective tissue disease patients among which 165 had ILD. The study population included a total of 147 RA patients (106 patients had no ILD and 41 patients had ILD). The study reported significantly higher KL-6 levels in RA patients with ILD compared to RA patients without ILD. Finally, Zheng et al., $2021^{29}$ also reported higher KL-6 levels in 24 Chinese RA patients with ILD compared to 26 RA patients without ILD.

To the best of our knowledge, no studies have evaluated KL-6 levels in RA patients with ILD in any African population, or in the Middle East. This is the first study to evaluate KL-6 levels in Egyptian RA patients with ILD.

We attempted to identify a cut-off for KL-6 for diagnosis of ILD. KL-6 level of $485 \mathrm{U} / \mathrm{mL}$ had an excellent sensitivity and specificity in discriminating between patients with ILD from patients without ILD. Zhong et al., 2020 ${ }^{24}$ performed a meta-analysis on the comparative diagnostic efficacy of KL-6 for CTD-ILD. The meta-analysis included a total of 23 articles covering 1265 patients with CTD-ILD and 1685 CTD patients without ILD. The study reported that apart from 2 studies with a cut-off value of around $900 \mathrm{U} / \mathrm{mL}$, the cut-off value from the remained studies approached to $500 \mathrm{U} / \mathrm{mL}$ which is rather close to the cut-off value deduced from ROC analysis in our study.

The presence of a UIP pattern on HRCT has been associated with poor prognosis and shorter survival time in RA patients with ILD compared to other CT patterns. ${ }^{30,31}$ In the present study, KL-6 levels were higher in patients with UIP compared to patients with other patterns of ILD. Similar results were reported by Kim et al., $2020{ }^{27}$ who described higher levels of KL-6 in 30 RA patients with UIP pattern of ILD compared to 54 RA patients with non-UIP pattern of ILD.

We divided the ILD patients into 4 groups according to the ILD grade by HRCT. KL- 6 levels were significantly higher in higher grades of ILD. Injury and regeneration of type 2 pneumocytes are characteristics of alveolitis occurring during ILD. $^{32}$ The main source of $\mathrm{KL}-6$ is the proliferating type 2 pneumocytes and serum KL6 levels reflect the degree of alveolar damage. ${ }^{16}$ Moreover, KL-6 was found to accelerate the proliferation of human fibroblasts and inhibit their apoptosis suggesting that increased levels of KL-6 may stimulate fibrotic processes in ILD. ${ }^{33}$ Such findings may explain the higher levels of KL-6 in higher grades of ILD observed in our study. Lee et al.,2019 23 also reported higher KL6 levels in higher CT grades, however, the study included patients from several connective tissue diseases and the authors did not compare KL-6 levels in various grades of ILD within each disease separately. Kinoshita et al., $2004^{26}$ also reported that KL-6 levels correlated with the severity ILD measured through chest HRCT. Moreover, Avouac et al., $2020^{20}$ reported higher KL-6 concentrations in RA-ILD patients with lesion extension $>15 \%$.

We attempted to identify cut-off levels for KL-6 to discriminate between different grades of ILD. KL-6 level of $940 \mathrm{U} / \mathrm{mL}$ had an excellent sensitivity and good specificity in discriminating grade 1 patients from the other 3 grades while KL-6 level of $1415 \mathrm{U} / \mathrm{mL}$ had good sensitivity and specificity in discriminating grade 1 and 2 patients from grade 3 and 4 patients. Regarding the discrimination between patients with grade 4 from the other 3 grades of ILD, the ROC curve revealed a cut-off of $1559 \mathrm{U} / \mathrm{mL}$, however as our study included only 4 patients with grade 4 ILD, this cut-off is inconclusive and further studies with larger number of RA patients with grade 4 ILD are needed to delineate a proper cut off.

Our study has a few limitations. Performance of KL-6 was not compared with pulmonary function tests. In addition, we did not follow up the patients to assess the role of $K L-6$ in prognosis assessment. Further prospective studies are needed to evaluate serial 
measurements of serum KL-6 level, PFT and chest HRCT to assess the role of serial KL- 6 measurement in monitoring ILD progression.

Among the added values of this study is that it is the first study to assess KL-6 in Egyptian RA patients with ILD, in addition to the included sample size of RA patients with ILD compared to previous studies. Studying the performance of potential biomarkers in different population provides more insight into the value of this biomarker.

In conclusion, we report higher levels of KL-6 in RA patients with ILD with higher levels in advanced stages of ILD compared to early stages suggesting $\mathrm{KL}-6$ as a circulating biomarker for estimating disease severity. Our study evaluated the diagnostic value of serum KL-6 levels for RA associated ILD. Our findings, in addition to previously published data in other populations, support that KL-6 may have a substantial role in evaluating ILD among RA patients.

\section{Author Contributions}

All authors made substantial contributions to the conception and design of the study. $\mathrm{RH}, \mathrm{NE}$ recruited patients and evaluated them clinically. DR performed the laboratory work. HS analyzed the HRCT. DR, RH and NE analyzed the data. DR, NE, RH drafted the manuscript and HS revised it critically.

\section{Declaration of Conflicting Interests}

The author(s) declared no potential conflicts of interest with respect to the research, authorship, and/or publication of this article.

\section{Funding}

The author(s) denies receipt of any financial support for the research, authorship, and/or publication of this article.

\section{Ethical approval}

The study protocol was reviewed and approved by The Ethical Committee, Faculty of Medicine, Ain Shams University (FMASU R 99, 2020).

\section{Informed consent}

Each patient provided a written informed consent prior to enrollment in the study.

\section{References}

1. Sivridis E, Kououpi $M$, Koukourakis $M$, et al. (2019). Rheumatoid nodules in thyroid gland parenchyma as an expression of rheumatoid arthritis: a case report. Journal of Medical Case Reports; 13: 159.

2. Gilles J. (2019). Extra-articular Manifestations and Comorbidity in Rheumatoid Arthritis: Potential Impact of Pre-Rheumatoid Arthritis Prevention. Clinical Therapeutics; 41(7): 1246-1255.

3. Alunno A, Gerli R, Giacomelli R, et al. (2017). Clinical, epidemiological, and histopathological features of respiratory involvement in rheumatoid arthritis. Biomed Res Int; 2017:7915340.

4. Vicente-Rabaneda E, Atienza-Mateo B, Blanco R, et al. (2021). Efficacy and safety of abatacept in interstitial lung disease of rheumatoid arthritis: $A$ systematic literature review. Autoimmun Rev; 20(6):102830.

5. Bendstrup R, Moller J, Kronborg-White S, et al. (2019). Interstitial Lung Disease in Rheumatoid Arthritis Remains a Challenge for Clinicians. J Clin Med; 8(12):2038.

6. Aguileria-Pickens G, Abud-Mendoza C. (2018). Pulmonary manifestations in systemic lupus erythematosus: pleural involvement, acute pneumonitis, chronic interstitial lung disease and diffuse alveolar hemorrhage. Rheumatol Clin; 14(5):194-300.

7. Perelas A, Silver R, Arrossi A, et al. (2020). Systemic sclerosis-associated interstitial lung disease. Lancet Respir Med; 8(3):304-320.

8. Hoffmann-Vold A, Allanore $\mathrm{Y}$, Alves $\mathrm{M}$, et al. (2021). Progressive interstitial lung disease in patients with systemic sclerosis- associated interstitial lung disease in the EUSTAR database. Annals of the Rheumatic Diseases; 80:219-227.

9. Sogkas G, Hirsch S, Olsson K, et al. (2020). Lung involvement in Primary Sjogren's syndrome- An under-diagnosed entity. Front Med; 7:332.

10. Hervier B, Uzunhan Y. (2019). Inflammatory Myopathy-Related Interstitial Lung Disease: From Pathophysiology to Treatment. Front Med; 6: 326.

11. Jang H, Yong S, Leem A, et al. (2021). Corticosteroid responsiveness in patients with acute exacerbation of interstitial lung disease admitted to the emergency department. Scientific Reports; 11:5762.

12. Chen J, Shi Y, Wang X, et al. (2013). Asymptomatic Preclinical Rheumatoid ArthritisAssociated Interstitial Lung Disease. Clin Dev Immunol; 2013: 406927. 
13. Bongartz T, Nannini C, Medina-Velasquez $\mathrm{Y}$, et al. (2010). Incidence and mortality of interstitial lung disease in rheumatoid arthritis: a populationbased study. Arthritis Rheum; 62(6):1583-1591.

14. Fessi R, Ourari B, Amar J, et al. (2018). Highresolution computed tomography fibrosis score and pulmonary function tests in interstitial lung disease: is there any correlation? European Respiratory Journal; 52(Suppl.62):PA2926.

15. Tzouvelekis A, Kouliatsis G, Anevalavis $S$, et al. (2005). Serum biomarkers in interstitial lung disease. Respiratory research; 6(1):78.

16. Ma H, Lu J, Song Y, et al. (2020). The value of serum Krebs von den lungen- 6 as a diagnostic marker in connective tissue disease associated with interstitial lung disease. BMC Pulmonary Medicine; 20:6.

17. Aletaha D, Neogi T, Silman A, et al. (2010). 2010 rheumatoid arthritis classification criteria: an American College of Rheumatology/ European League Against Rheumatism collaborative initiative. Ann Rheum Dis; 69(9): 1580-1588.

18. Aletaha D, Neogi T, Silman A, et al. (2010). 2010 rheumatoid arthritis classification criteria: an American College of Rheumatology/ European League Against Rheumatism collaborative initiative. Arthritis Rheumatol; 62(9): 2569-2581.

19. Volkmann E, Tashkin D, Kuwana M, et al. (2019). Progression of Interstitial Lung Disease in Systemic Sclerosis: The Importance of Pneumoproteins Krebs von den Lungen 6 and CCL18. Arthritis Rheumatol; 71(12):2059-2067.

20. Avouac J, Cauvet A, Steelandt A, et al. (2020). Improving risk-stratification of rheumatoid arthritis patients for interstitial lung disease. PLOS One; 15(5): e0232978.

21. d'Alessandro $M$, Bergantini $L$, Cameli $P$, et al. (2020). Krebs von den Lungen-6 as a biomarker for disease severity assessment in interstitial lung disease: a comprehensive review. Biomark Med; 14(8):665-674.

22. Qin H, Xu X, Zou J, et al. (2019). Krebs von den Lungen- 6 associated with chest high-resolution CT score in evaluation severity of patients with interstitial lung disease. Pulmonology; 25(3):143148.

23. Lee J, Lee E, Ha Y, et al. (2019). Serum KL-6 levels reflect the severity of interstitial lung disease associated with connective tissue disease. Arthritis Res Ther; 21(1): 58.

24. Zhong D, Wu, C, Bai J, et al. (2020). Comparative diagnostic efficacy of serum Krebs von den Lungen- 6 and surfactant $D$ for connective tissue disease-associated interstitial lung diseases. A meta-analysis. Medicine; 99(16): e19695.

25. Cao X, Hu S, Xu D, et al. (2019). Serum levels of Krebs von den Lungen- 6 as a promising marker for predicting occurrence and deterioration of systemic sclerosis-associated interstitial lung disease from a Chinese cohort. Int J Rheum Dis; 22(1):108-115.

26. Kinoshita F, Hamano $H$, Harada $H$, et al. (2004). Role of KL- 6 in evaluating the disease severity of rheumatoid lung disease: comparison with HRCT. Respir Med; 98(11):1131-1137.

27. Kim H, Choi K, Jacob J, et al. (2020). Prognostic role of blood KL-6 in rheumatoid arthritisassociated interstitial lung disease. PLoS One; 15(3): e0229997.

28. Tanaka N, Nishimura K, Waki D, et al. (2021). Annual variation rate of $\mathrm{KL}-6$ for predicting acute exacerbation in patients with rheumatoid arthritis-associated interstitial lung disease. Modern Rheumatology; 10.1080/14397595.2021. 1879346.

29. Zheng M, Lou A, Zhang $H$, et al. (2021). Serum KL6, CA 19.9, CA 125 and CEA are diagnostic biomarkers for rheumatoid arthritis-associated interstitial lung disease in the Chinese population. Rheumatology and Therapy; 8: 517527.

30. Solomon J, Chung J, Cosgrove G, et al. (2016). Predictors of mortality in rheumatoid arthritis associated interstitial lung disease. Eur Respir J; 47:588-596.

31. Kim H, Lee J, Lee E, et al. (2020). Risk prediction model in rheumatoid arthritis-associated interstitial lung disease. Respirology; 25(12):1257-1264.

32. Ishikawa N, Hattori N, Yokoyama A, et al. (2012). Utility of KL-6/MUC1 in the clinical management of interstitial lung diseases. Respir Investig; 50(1):3-13.

33. Ohshimo S, Yokoyama A, Noboru H, et al. (2005). $\mathrm{KL}-6$, a human MUC-1 mucin, promotes proliferation and survival of lung fibroblasts. Communications; 338(4):1845-1852. 